\title{
Efficiency of transmission of discrete messages in a residue number system
}

\author{
N.V. Zakharchenko ${ }^{a}$, M.M. Hajiyev ${ }^{a}$, T.M. Mansurov ${ }^{b *}$, A.A. Nazarenko ${ }^{a}$, \\ N.S. Salmanov ${ }^{b}$
}

${ }^{a}$ Odessa State University of Information Technologies and Communications, Odessa, Ukraine

${ }^{b}$ Azerbaijan Technical University, Baku, Azerbaijan

\begin{tabular}{|c|c|}
\hline $\begin{array}{l}\text { A R T I C L E I N F O } \\
\end{array}$ & A B S T RACT \\
\hline $\begin{array}{l}\text { Article history: } \\
\text { Received 10.10.2021 } \\
\text { Received in revised form } 16.10 .2021 \\
\text { Accepted } 27.10 .2021 \\
\text { Available online } 29.12 .2021\end{array}$ & $\begin{array}{l}\text { The authors analyze the process of transmission of discrete mes- } \\
\text { sages in a residue number system, i.e., the efficiency of the repre- } \\
\text { sentation of decimal numbers } N_{10} E 1 / 105 \text { in a residue number } \\
\text { system for different values of the module, that is, } P_{1}=3 ; P_{2}=5 \text {; } \\
P_{3}=7 \text {. It is noted that it is characteristic of modern information }\end{array}$ \\
\hline $\begin{array}{l}\text { Keywords: } \\
\text { Entropy } \\
\text { Residue number system } \\
\text { Timer signal construction } \\
\text { Discrete message } \\
\text { Information parameter } \\
\text { Nyquist element }\end{array}$ & $\begin{array}{l}\text { transmission systems that the information is contained in the in- } \\
\text { formation parameter at a fixed interval of the Nyquist element. } \\
\text { The increase in the capacity is achieved by increasing the num- } \\
\text { ber of states of the information parameter in this interval, i.e., the } \\
\text { number of different amplitudes, phases and frequencies of the } \\
\text { carrier oscillation. In addition, using a residue number system } \\
\text { when representing decimal numbers } N_{10} E 1 / 105 \text {, the sum of the } \\
\text { digits of individual numbers in most cases is less than } 10 \text { (except } \\
\text { for the number N104) and an increase in the probability of not } \\
\text { using moduli } P_{i} E 3 ; 5 ; 7 \text { leads to an increase in the entropy of } \\
\text { the corresponding events. }\end{array}$ \\
\hline
\end{tabular}

\section{Introduction}

As is known [1], two types of basic functions are distinguished in communication theory, and most often these functions include report functions and pulse functions. A feature of the first of them is the time dependence of their values. When building ultra-reliable systems, a redundancy method is used, in which reliability is ensured by redundancy, an example of which is redundancy (correcting) codes [2], in which several verifying code elements synthesized on the basis of information ones are added to the information code elements. Arithmetic codes of the second class have broader correcting properties, which not only allow to use control elements more efficiently, but also to verify the number itself.

The main purpose of this article is to analyze the process of transmitting discrete messages in a residue number system. In this regard, we analyze of the efficiency of the representation of decimal numbers $N_{10} E 1 / 105$ in a residue number system for different values of the module, i.e., $P_{1}=$ $3 ; P_{2}=5 ; P_{3}=7[3]$.

\footnotetext{
${ }^{*}$ Corresponding author.

E-mail addresses: onat@onat.edu.ua (N.V. Zakharchenko), gadjievmm@ukr.net (M.M. Hajiyev), tofiqmansurov@rambler.ru (T.M. Mansurov), onat@onat.edu.ua (A.A. Nazarenko), nariman_s@box.az (N.S. Salmanov). 


\section{The state of the art}

The main ideas of synthesizing the efficiency of transmission of discrete messages in a residue number system were formulated in the works of N.V. Zakharchenko, D.Ye. Vakman, L.E. Varakin, O.D. Viterts, T.M. Mansurov and others. A significant contribution to the development of this area was made by the scientific schools of P.F. Polyakov, V.I. Khakhanov, M.B. Sverdlik and V.G. Koshevoy.

The foundation of the study of transmission systems with narrowband signals was laid by $\mathrm{H}$. Nyquist. This study was further developed in the articles and monographs by D. Middleton, O.O. Markevich, L.M. Fink and others. O.G. Zyuko, V.A. Kisel, N.V. Zakharchenko, E.M. Rudoy, P.P. Vorobienko, Yu.I. Losev, V.V. Popovsky, V.K. Steklov, T.M. Mansurov and others made a considerable contribution to solving problems of enhancing the efficiency of methods for signal transmission and processing.

The main technical resources of information transmission systems is the energy excess of the signal over the noise at the receiver input. The technical effect of the operation of information transmission systems is determined by the quantity and quality of the transmitted information, i.e., the transmission rate and the probability of correct reception.

A characteristic feature of modern information transmission systems and works by the above authors is that the information is contained in the information parameter at a fixed interval of the Nyquist element. The increase in the capacity is achieved by increasing the number of states of the information parameter in this interval, i.e., the number of different amplitudes, phases and frequencies of the carrier oscillation.

In this article, due to changes in the restrictions on the lifetime of the basic element of the signal construction, an increase in the channel capacity is obtained by reducing the energy distance between the signal constructions. In this case, the information parameter in these constructions is not the parameters of the current or voltage, but the parameters of time. Such signals, by the definition of G.E. Pukhov and V.F. Bardachenka is called timer signals. The timer signal construction contains several time intervals, the length of which is not less than, but not multiple of, the Nyquist element. The information in the timer signal construction is contained in the durations of these segments and their relative position.

\section{Features of the formation of Table 1 [3]}

1. Total code word numbers $N_{10} \frac{E 1}{105}$;

2. Each number $N_{10} E 1 / 105$ is represented by three residues in $P_{i}$ E $3 ; 5 ; 7$;

3. Each of the three moduli $3 ; 5 ; 7$ is used to determine the residues of the division of next number № - 1 / 105 by the corresponding moduli $P_{i}$ E 3; 5; 7;

4. Each of the moduli $P_{i} \mathrm{E} 3 ; 5 ; 7$ is used to form the residues of the moduli:

$$
\begin{gathered}
P_{1}=3 ; \text { residues } 1 ; 2 ; 0 ; \\
P_{2}=5 ; \text { residues } 1 ; 2 ; 3 ; 4 ; 0 ; \\
P_{3}=7 ; \text { residues } 1 ; 2 ; 3 ; 4 ; 5 ; 6 ; 0 .
\end{gathered}
$$

5. The residues indicated in 4 are repeated to be written under the next decimal numbers [3]. In this case, the residues have the following values:

$$
\begin{aligned}
& \mathrm{P}(3) \text { repeat } 35 \text { times }(105 / 3=35) \text {; } \\
& \mathrm{P}(5) \text { repeat } 21 \text { times }(105 / 5=21) \text {; } \\
& \mathrm{P}(7) \text { repeat } 15 \text { times }(105 / 7=15) .
\end{aligned}
$$


6. After completing 4 and 5, under each of the 105 numbers, we mark (vertically) individual numbers from the residues of 4 .

7. The sum of the digits of the three residues " $S$ " with the changed number 10 , varies from one to tens. The largest sum " $S$ " of three residues is $S=2+4+6=12$ for number 104 (Table 1 ).

Table 1

\begin{tabular}{|c|c|c|c|c|c|c|c|c|c|c|c|c|c|c|c|c|c|c|c|c|c|}
\hline$N_{i}$ & 1 & 2 & 3 & 4 & 5 & 6 & 7 & 8 & 9 & 10 & 11 & 12 & 13 & 14 & 15 & 16 & 17 & 18 & 19 & 20 & 21 \\
\hline 3 & 1 & 2 & 0 & 1 & 2 & 0 & 1 & 2 & 0 & 1 & 2 & 0 & 1 & 2 & 0 & 1 & 2 & 0 & 1 & 2 & 0 \\
\hline 5 & 1 & 2 & 3 & 4 & 0 & 1 & 2 & 3 & 4 & 0 & 1 & 2 & 3 & 4 & 0 & 1 & 2 & 3 & 4 & 0 & 1 \\
\hline 7 & 1 & 2 & 3 & 4 & 5 & 6 & 0 & 1 & 2 & 3 & 4 & 5 & 6 & 0 & 1 & 2 & 3 & 4 & 5 & 6 & 0 \\
\hline$N_{i}$ & 22 & 23 & 24 & 25 & 26 & 27 & 28 & 29 & 30 & 31 & 32 & 33 & 34 & 35 & 36 & 37 & 38 & 39 & 40 & 41 & 42 \\
\hline 3 & 1 & 2 & 0 & 1 & 2 & 0 & 1 & 2 & 0 & 1 & 2 & 0 & 1 & 2 & 0 & 1 & 2 & 0 & 1 & 2 & 0 \\
\hline 5 & 2 & 3 & 4 & 0 & 1 & 2 & 3 & 4 & 0 & 1 & 2 & 3 & 4 & 0 & 1 & 2 & 3 & 4 & 0 & 1 & 2 \\
\hline 7 & 1 & 2 & 3 & 4 & 5 & 6 & 0 & 1 & 2 & 3 & 4 & 5 & 6 & 0 & 1 & 2 & 3 & 4 & 5 & 6 & 0 \\
\hline$N_{i}$ & 43 & 44 & 45 & 46 & 47 & 48 & 49 & 50 & 51 & 52 & 53 & 54 & 55 & 56 & 57 & 58 & 59 & 60 & 61 & 62 & 63 \\
\hline 3 & 1 & 2 & 0 & 1 & 2 & 0 & 1 & 2 & 0 & 1 & 2 & 0 & 1 & 2 & 0 & 1 & 2 & 0 & 1 & 2 & 0 \\
\hline 5 & 3 & 4 & 0 & 1 & 2 & 3 & 4 & 0 & 1 & 2 & 3 & 4 & 0 & 1 & 2 & 3 & 4 & 0 & 1 & 2 & 3 \\
\hline 7 & 1 & 2 & 3 & 4 & 5 & 6 & 0 & 1 & 2 & 3 & 4 & 5 & 6 & 0 & 1 & 2 & 3 & 4 & 5 & 6 & 0 \\
\hline$P_{i}$ & 64 & 65 & 66 & 67 & 68 & 69 & 70 & 71 & 72 & 73 & 74 & 75 & 76 & 77 & 78 & 79 & 80 & 81 & 82 & 83 & 84 \\
\hline 3 & 1 & 2 & 0 & 1 & 2 & 0 & 1 & 2 & 0 & 1 & 2 & 0 & 1 & 2 & 0 & 1 & 2 & 0 & 1 & 2 & 0 \\
\hline 5 & 4 & 0 & 1 & 2 & 3 & 4 & 0 & 1 & 2 & 3 & 4 & 0 & 1 & 2 & 3 & 4 & 0 & 1 & 2 & 3 & 4 \\
\hline 7 & 1 & 2 & 3 & 4 & 5 & 6 & 0 & 1 & 2 & 3 & 4 & 5 & 6 & 0 & 1 & 2 & 3 & 4 & 5 & 6 & 0 \\
\hline$N_{i}$ & 85 & 86 & 87 & 88 & 89 & 90 & 91 & 92 & 93 & 94 & 95 & 96 & 97 & 98 & 99 & 100 & 101 & 102 & 103 & 104 & 105 \\
\hline 3 & 1 & 2 & 0 & 1 & 2 & 0 & 1 & 2 & 0 & 1 & 2 & 0 & 1 & 2 & 0 & 1 & 2 & 0 & 1 & 2 & 0 \\
\hline 5 & 0 & 1 & 2 & 3 & 4 & 0 & 1 & 2 & 3 & 4 & 0 & 1 & 2 & 3 & 4 & 0 & 1 & 2 & 3 & 4 & 0 \\
\hline 7 & 1 & 2 & 3 & 4 & 5 & 6 & 0 & 1 & 2 & 3 & 4 & 5 & 6 & 0 & 1 & 2 & 3 & 4 & 5 & 6 & 0 \\
\hline
\end{tabular}

\section{Synthesis of transmission of code words over a communication channel based on Table 1}

Taking into account table 1, the features of its structure should be noted [3]:

1. The residues $\mathrm{O}$ of each of the moduli $P_{i} \mathrm{E} 3 ; 5 ; 7$ have a different number of decimal digits:

$$
\begin{gathered}
P_{1}=3 \text {--- } 0_{1}--1 ; 2 ; 0 ; \\
P_{2}=5 \text {--- } 0_{1} \text {-- } 1 ; 2 ; 3 ; 4 ; 0 ; \\
P_{3}=7 \text {--- } 0_{1}--1 ; 2 ; 3 ; 4 ; 5 ; 6 ; 0 .
\end{gathered}
$$

2. It is technologically inefficient to encode a code construction when transmitting residues of different duration, i.e., the code words will have different lengths.

3. The representation of each decimal number №10 in the form of three residues of the specified moduli is positive in the structure of the table:

$$
\begin{aligned}
& P_{1}=3 ; \\
& P_{2}=5 ; \\
& P_{3}=7 .
\end{aligned}
$$

4. Among all the residues, for various moduli of the inputs of the residues $\mathrm{O}$ and with this residue, the Nyquist element should be synthesized during transmission [2];

5. The digits in the residues differ from $\mathrm{O}$, must correspond to the realities of the transmitted signal segments:

$$
P_{1}=3--t_{o}+0 * \Delta ; t_{o}+1 * \Delta ; t_{o}+2 * \Delta ;
$$




$$
\begin{gathered}
P_{2}=5--t_{o}+0 * \Delta ; t_{o}+1 * \Delta ; t_{o}+2 * \Delta ; t_{o}+3 * \Delta ; t_{o}+4 * \Delta ; \\
P_{3}=7--t_{o}+0 * \Delta ; t_{o}+1 * \Delta ; t_{o}+2 * \Delta ; t_{o}+3 * \Delta ; t_{o}+4 * \Delta ; t_{o}+5 * \Delta ; t_{o}+6 * \Delta .
\end{gathered}
$$

6. From 4, the longest code word consists of: №10 --- 104;

$$
\mathrm{T}_{\mathrm{M}}=3 \mathrm{~T}_{\mathrm{o}}+2 \Delta+4 \Delta+6 \Delta=3 \mathrm{~T}_{\mathrm{o}}+12 \Delta .
$$

7. If we assume that the length of the Nyquist element $t_{o}=\frac{1}{\Delta F}$ consists of five elements $5 \Delta$, then $\Delta=\frac{t_{o}}{5}=0,2 t_{o}$ and the expression will take the form:

$$
T_{m}=3 t_{o}+12 \Delta=3 t_{o}+12 * 0,2 t_{o}=5,4 t_{o} .
$$

8. Estimate the transmission time of 105 code words in positional coding, then the number of binary elements " $n$ " will be equal to:

$$
n-E+\log _{2} N 10=E * \log _{2} 105=7 .
$$

Comparing the maximum length of a code word with a length of $n=7$, we see that the time loss during coding in residues, the transmission time is less in comparison with positional coding.

\section{Message entropy in coding in a residue number system [4]}

The message entropy for an unequiprobable alphabet is [5]:

$$
H=-\sum_{i=1}^{m} P_{i} \log _{2} P_{i} .
$$

Since three moduli $P_{1}=3 ; P_{2}=5 ; P_{3}=7$ with recurrency frequency $\mathrm{P}(3)$ - 35 times; $\mathrm{P}(5)$ 21 times; $\mathrm{P}(7)$ - 15 times are used in Table 1, the probabilities of them being used are:

$$
\begin{aligned}
& P_{1}=\frac{35}{105}=\frac{1}{3} \\
& P_{2}=\frac{21}{105}=\frac{1}{5} ; \\
& P_{3}=\frac{15}{105}=\frac{1}{7} .
\end{aligned}
$$

Therefore, the message entropies will be:

$$
\begin{aligned}
H_{1} & =\frac{1}{3} \log _{2} \frac{1}{3}=\frac{1}{3} \log _{2} 1-\log _{2} 3=1,58 ; \\
H_{2} & =\frac{1}{5} \log _{2} \frac{1}{5}=\frac{1}{5} \log _{2} 1-\log _{2} 5=2,32 ; \\
H_{3} & =\frac{1}{7} \log _{2} \frac{1}{7}=\frac{1}{7} \log _{2} 1-\log _{2} 7=2,8 .
\end{aligned}
$$

It follows from the values of entropy that with a decrease in the probability of using the moduli $P_{i}: P_{1}<P_{2}<P_{3}$, the entropy of the event increases.

\section{Conclusion}

Thus, it is characteristic of modern information transmission systems that the information is contained in the information parameter at a fixed interval of the Nyquist element. The increase in the capacity is achieved by increasing the number of states of the information parameter in this interval, i.e., the number of different amplitudes, phases and frequencies of the carrier oscillation. In addition, it can be noted that using of a residue number system when representing decimal numbers $N_{10} E 1 / 105$ the sum of the digits of individual numbers in most cases is less than 10 (except for the 
number N104) and an increase in the probability of not using the moduli $P_{i}$ E $3 ; 5 ; 7$ leads to an increase in the entropy of the corresponding events.

\section{References}

[1] V.A. Ignatov, Theory of information and signal transmission, V.A. Ignatov, M., Sov. Radio, (1979) 280 p.

[2] N.N. Buga, Fundamentals of the theory of communication and data transmission, N.N. Buga, L. Vika I.M. A.F. Mozhaisky, (1968) 542 p.

[3] V.A. Torgashev, System of residual classes and reliability of communication, V.A. Torgashev, M., Sov. Radio, (1973) $270 \mathrm{p}$.

[4] M.V. Zakharenko, Systems of transmission of tributes, Efficiency of block code, M.V. Zakharenko, V.Ya. Kildishev et al., Odesa, (2014) 487 p.

[5] N.V. Zakharenko, Informational parameters of positional and timer codes, Volume 1 Information parameters of positional codes, N.V. Zakharenko, S.M. Gorokhov, A.V. Kochetkov, Odessa, (2018) 211 p. 